\title{
Comparison of the Efficacy of Conventional Echocardiographic Parameters in the Diagnosis of Significant Coronary Artery Stenosis
}

\author{
Xiao Zhi Zheng ${ }^{1}$; Bin Yang ${ }^{2, *}$; Jing Wu ${ }^{1}$ \\ ${ }^{1}$ Department of Ultrasound, Affiliated Hospital of Nantong University (The First People's Hospital of Yancheng), Yancheng, P.R China \\ ${ }^{2}$ Department of Ultrasound, Jinling Hospital, Nanjing University School of Medicine, Nanjing City, P.R China \\ ${ }^{*}$ Corresponding author: Bin Yang, Department of Ultrasound, Jinling Hospital, Nanjing University School of Medicine, Nanjing City, P.R China. Tel: +8625-80861314, Fax: +8625- \\ 80863136, E-mail:yb12yx@Hotmail.com
}

Received: April 5, 2013; Revised: June 2, 2013; Accepted: June 11, 2013

\begin{abstract}
Background: For diagnosis of significant coronary artery stenosis, the most accurate parameter among the conventional echocardiographic parameters remains unknown.

Objectives:To assess the diagnostic efficacy of conventional echocardiographic parameters in the diagnosis of significant coronary artery stenosis and their correlation with the percentage of stenosis considering conventional coronary angiography as gold standard.

Patients and Methods: Seventy eight individuals were included in the study. The three echocardiographic parameters including the coronary sinus flow [flow (mL/minute)], the global left ventricular perfusion by dividing the coronary sinus flow by left ventricular mass [flow/LVM (mL/minute)] and the difference between the left ventricular mass at end diastole and peak systole using area-length calculation methods $[\mathrm{LV}(\mathrm{d}-\mathrm{s})$ mass A-L $]$ were assessed using receiver operating characteristic (ROC) analysis for their accuracy to distinguish between normal subjects and patients with significant $(>50 \%)$ coronary artery disease (CAD).

Results: Flow (mL/minute), flow/LVM (mL/minuteg) and LV (d-s) mass A-L all correlated significantly with the percentage of stenosis ( $\mathrm{r}$ $=-0.64, \mathrm{P}<0.001 ; \mathrm{r}=-0.47, \mathrm{P}<0.001 ; \mathrm{r}=-0.56, \mathrm{P}<0.001$, respectively). With the use of the percentage of stenosis $>50 \%$ as the criteria to distinguish patients with or without CAD, the areas under the ROC curve for flow (mL/minute) were 0.75 , while they were 0.57 for flow/LVM (mL/minuteg) and 0.59 for LV(d-s) mass A-L. The percentage of stenosis $>70 \%$ was best detected by coronary sinus flow $<198 \mathrm{~mL} / \mathrm{minute}$ (sensitivity, 81.35\%; specificity, 70.37\%; positive predictive value, 63.63\%; negative predictive value, $86.36 \%$ and accuracy, $75 \%$; $\mathrm{P}<0.001$ ). Conclusions: The coronary sinus flow per minute is the most accurate parameter among the three echocardiographic parameters mentioned above for the assessment of significant stenosis of the coronary artery.
\end{abstract}

Keywords:Coronary Sinus Flow; Myocardial Perfusion; Left Ventricular Mass; Coronary Artery Stenosis

\section{Background}

The assessment of the coronary artery stenosis by conventional echocardiography remains challenging due to inadequate visualization of the coronary arteries, especially for the mid and distal parts (1-3). As $95 \%$ of the left ventricular perfusion drains to the right atrium through the coronary sinus (4), the flow in this vessel is a good representation of the global left ventricular perfusion. Over the last decade, coronary flow reserve in the coronary sinus has been used to diagnose the significant coronary artery stenosis by transesophageal Doppler echocardiography (TEE). TEE is a semi-invasive procedure and the vasodilator drugs, such as adenosine, are not always harmless. A recent study demonstrates that reduced antegrade flow in the coronary sinus detected by transthoracic Doppler echocardiography (TTE) is a sensitive predictor of coronary artery stenosis in hypertensive patients (5). Global left ventricular perfusion calculated by dividing coronary sinus flow by the left ventricular mass (LVM) is another parameter for the assessment of significant stenosis of the coronary artery
(6). Moreover, in clinic, we have also found that the difference of LVM at end diastole and peak systole using area-length calculation methods [LV (d-s) mass A-L] correlated significantly with the mean percentage of stenosis in patients with CAD, and the reduced LV (d-s) mass is a sensitive and specific predictor of significant stenosis of the left coronary artery territory (7). The question is "among the three mentioned parameters of coronary sinus flow, the global left ventricular perfusion and the difference of the left ventricular mass in the cardiac cycle, which is the most accurate parameter?" it remains unknown.

\section{Objectives}

The purpose of this study was to compare the efficacy of the above mentioned echocardiographic parameters in the diagnosis of significant coronary artery stenosis using coronary angiography as gold standard.

Copyright (C) 2015, Tehran University of Medical Sciences and Iranian Society of Radiology. This is an open-access article distributed under the terms of the Creative Commons Attribution-NonCommercial 4.0 International License (http://creativecommons.org/licenses/by-nc/4.0/) which permits copy and redistribute the material just in noncommercial usages, provided the original work is properly cited. 


\section{Patients and Methods}

\subsection{Study Population}

Our study was approved by the local human research ethics committee and free informed consent was obtained from all the participants. The study population consisted of 78 participants who had undergone coronary angiography because of suspected CAD. Thirty-eight of these participants were normal participants (mean age, $55.9 \pm 10.2$ years; range, $45-66$ years) and 40 were CAD patients who had more than $50 \%$ stenosis in coronary angiography (mean age, 54.9 \pm 11.8 years; range, $43-66$ years). All the participants had a sinus rhythm, normal systolic function of the left and right ventricles, normal right ventricular systolic pressure, normal right atrial pressure and normal pulmonary artery pressure, which was confirmed by right heart catheterization. The participants with hypertension, congenital heart disease, valvular disease, cardiomyopathy, diabetes mellitus, and intracoronary shunts were excluded from the study.

\subsection{Echocardiographic Measurements}

The echocardiographic study was performed in all participants while they were lying in the left lateral decubitus position and the electrocardiography was being recorded simultaneously. The echocardiographic data were acquired using a commercially available ultrasonic system: Vivid E9 ultrasound machine (GE Healthcare, Horten, Norway) equipped with M5S single-crystal matrix-array transducer. All acquisitions were performed by two experienced operators who were blinded to all clinical data and previous reading. Data sets were stored digitally for off-line assessment and analysis. All values for each parameter were obtained by averaging measurements from three successive cardiac cycles. First of all, the standard parasternal long axis view was obtained. An M-mode tracing ( $66.7 \mathrm{~mm} / \mathrm{s}$ ) was recorded for the subsequent measurements: diastolic interventricular septal thickness (IVST), left ventricular diastolic posterior wall thickness (LVPWT), left ventricular end-diastolic diameter (LVEDD), and left ventricular end-systole diameter (LVESD). Then the left ventricular ejection-fraction (LVEF) was automatically calculated by the ultrasonic system. The left ventricular mass (LVM) was derived using the following formula:

LVM $($ M-mode $)(\mathrm{g})=0.8 \times\left[1.04 \times(\text { IVST }+ \text { LVEDD }+ \text { LVPWT })^{3}\right.$ - LVEDD $\left.^{3}\right]+0.6$.

The formula has been anatomically validated by Devereux (8). Second, the parasternal right ventricular inflow tract view was obtained. The transducer was manipulated to visualize the mouth of the coronary sinus (Figure $1 \mathrm{~A}$ ). To avoid the influence of atrial contraction, the coronary sinus diameter (Dcs) was measured at a 1 $\mathrm{cm}$ distance from the mouth in the end diastolic phase before the P wave on ECG using adjust M-mode tracing (Figure $1 \mathrm{~B}$ ). A pulsed-wave sample volume (3 $\mathrm{mm}$ ) was placed at a $1 \mathrm{~cm}$ distance from the mouth, and then rotated by a small amount (Doppler angle between the ultrasound beam and vessels $<30^{\circ}$ ) to obtain the optimum Doppler flow signals, and spectral recordings of the flow were made. The velocity time integral (VTI, cm) were determined through digitized Doppler spectral envelopes (Figure $1 \mathrm{C}$ and D). The coronary sinus flow (Flow) per minute was calculated according to the formula:

Flow per minute $(\mathrm{mL} /$ minute $)=\pi \times \mathrm{D}^{2} / 4 \times \mathrm{VTI} \times$ heart rate (HR),

where $\pi$ is the ratio of the circumference of a circle to its diameter, and $\mathrm{D}$ is the diameter of the coronary sinus (1). The average value of three spectral and planimetric envelopes was used. Global left ventricular perfusion $(\mathrm{mL} /$ minute $g$ ) $=$ the flow per minute/LVM (M-mode) (3).

Third, the apical two-chamber view was obtained. Left ventricular long axis length at end diastole (LVLd apical) and at peak systole (LVLs apical) were measured from the endocardial boundaries to the middle of the mitral annulus (Figure $2 \mathrm{~A}$ and C). Finally, the left ventricular short axis view at the papillary muscle level was obtained. Left ventricular epicardial short axis area the level of the papillary muscle tips at end diastole (LVAd SAX EPI), left ventricular endocardial short axis area at papillary muscle level at end diastole (LVAd SAX PM); left ventricular epicardial short axis area at the level of the papillary muscle tips at peak systole (LVAs SAX EPI) and left ventricular endocardial short axis area at papillary muscle level at peak systole (LVAs SAX PM) were measured in the order mentioned. Left ventricular mass (area-length) at end diastole (LVd mass A-L) and left ventricular mass (arealength) at peak systole (LVs mass A-L) were automatically calculated by the ultrasonic system (Figure $2 \mathrm{~B}$ and D). LV (d-s) mass A-L = (LVd mass A-L) - (LVs mass A-L).

\subsection{Coronary Angiography}

Invasive coronary angiography was performed in all participants through the femoral approach within 24 hours of TTE according to the standard method of Judkins (9) using the COROSKOP plus angiographic complex (Siemens AG, Berlin, Germany) and standard catheters and conventional views. Coronary diameter stenosis of $>$ $50 \%$ in coronary angiography was considered as significant $(10,11)$. The number of coronary arteries with significant stenosis, the localization of stenosis and the maximal percentages of stenosis were determined.

\subsection{Reproducibility}

Intraobserver variability was assessed in 23 participants by repeating the measurements on two occasions (3 days apart) under the same basal conditions. To test the interobserver variability, the measurements were performed on the same subject by a second blinded observer. Variability was calculated as the mean percentage error, derived as the difference between the two sets of measurements, and divided by the mean observations. 

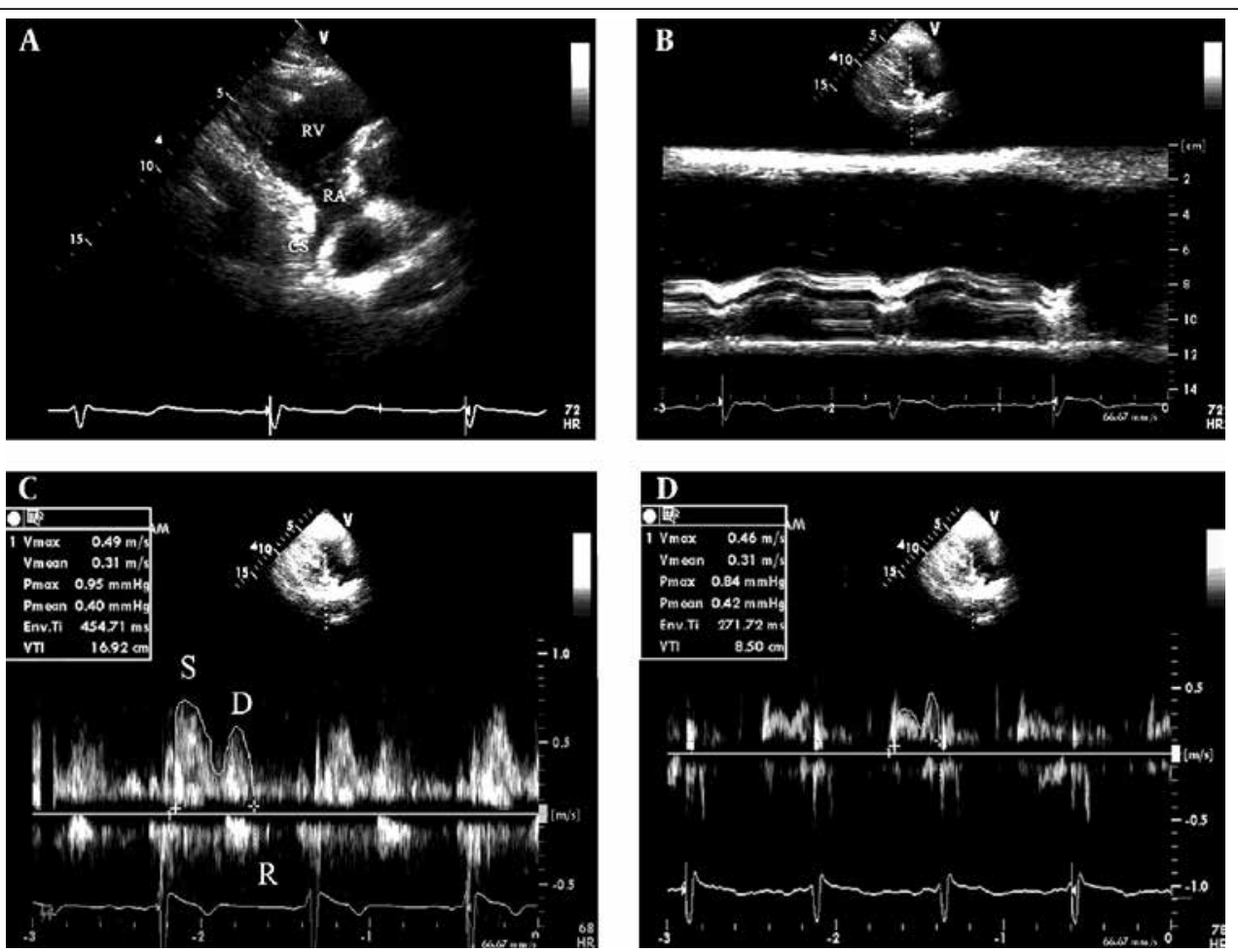

Figure 1. A, The coronary sinus seen from the parasternal right ventricular inflow tract view and B, the measurement of the diameter of the coronary sinus using adjust M-mode ultrasonography; $\mathrm{C}$, the Doppler spectrum of coronary blood flow in the coronary sinus obtained from the parasternal right ventricular inflow tract view and the measurement of Doppler parameters of the coronary sinus flow by digitized Doppler spectral envelops in normal subjects and D, patients with coronary artery disease. RA, right atrium; RV, right ventricle; CS, coronary sinus; S, systolic wave of the antegrade blood flow;

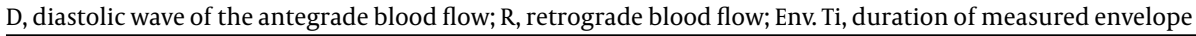
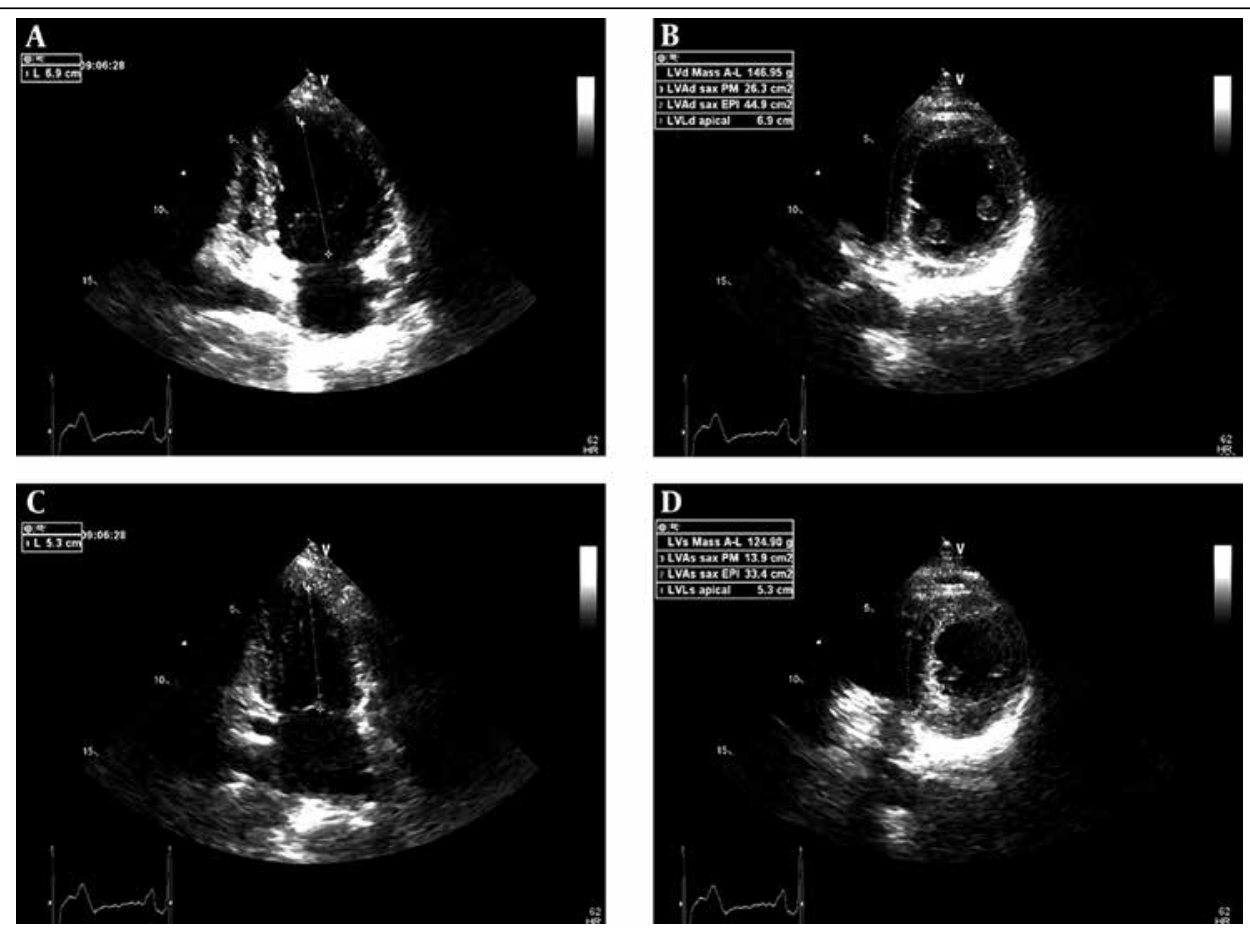

Figure 2. The measurements of left ventricular mass (area-length) at end diastole and peak systole using biplane two-dimensional echocardiographic methods. A, LVLd apical, left ventricular length at end diastole, apical; B, LVAd SAX EPI, left ventricular epicardial short axis area at the level of the papillary muscle tips at end diastole; B, LVAd SAX PM, left ventricular endocardial short axis area at papillary muscle level at end diastole; C, LVLs apical, left ventricular length at peak systole, apical; D, LVAs SAX EPI, left ventricular epicardial short axis area at the level of the papillary muscle tips at peak systole; D, LVAs SAX PM, left ventricular endocardial short axis area at papillary muscle level at peak systole 


\subsection{Statistical Analysis}

Data were expressed as the mean \pm SD. The differences between the two groups were tested using an unpaired two tailed t test. Flow (mL/minute), flow/LVM (mL/ minuteg) and LV (d-s) mass A-L were compared with the mean percent stenosis diameter by linear correlation analysis. A receiver operating characteristic curve (ROC) analysis was used to compare the performance of these parameters mentioned above in discrimination between patients with or without CAD. Discriminant analysis was conducted in the traditional manner. A value of $\mathrm{P}<0.05$ was considered statistically significant. Statistical analysis was performed with SPSS version 13 software for Windows (SPSS Inc, Chicago, IL).

\section{Results}

\subsection{Echocardiographic Parameters}

As shown in Table 1 and Table 2, HR, SBP, DBP, IVST, LVPWT,
LVEDD, LVEF and LVd mass A-L did not differ between the normal participants and patients with CAD and among the single-vessel, double-vessel and triple-vessel lesions in patients with CAD. Compared with normal participants, the patients with CAD had significantly greater PP, LVM (M-mode), LVs mass A-L and had significantly smaller Dcs, significantly lower VTI, flow, flow/LVM and LV (d-s) mass A-L $(\mathrm{g})(\mathrm{P}<0.05)$. Compared to patients with singlevessel and double-vessel stenosis, patients with triple vessel stenosis had significantly lower Dcs, VTI, flow, and flow/LVM $(\mathrm{P}<0.05)$.

\subsection{Coronary Angiographic Findings}

Twelve cases were single vessel disease, 16 cases were two-vessel disease and 12 cases were three-vessel disease (Table 2). The culprit coronary arteries where the stenotic lesions were located are shown in Table 3. Sixty-five lesions were more than $50 \%$ stenosis and 70 lesions were more than $70 \%$ stenosis. The mean percent stenosis diameter in the left coronary artery territory was $68.36 \pm 17.97 \%$.

Table 1. Clinical and Echocardiographic Parameters of Normal Subjects and Patients with Coronary Artery Disease ${ }^{\text {a }}$

\begin{tabular}{|c|c|c|}
\hline Parameters & Normal Subjects & Patients with CAD \\
\hline \multicolumn{3}{|l|}{ Clinical Parameters } \\
\hline HR, beats/min & $72.51 \pm 11.84$ & $71.33 \pm 10.89$ \\
\hline SBP, mmHg & $119 \pm 20.79$ & $125.44 \pm 23.31$ \\
\hline DBP, mmHg & $75.21 \pm 6.64$ & $73.75 \pm 8.89$ \\
\hline $\mathrm{PP}, \mathrm{mmHg}$ & $40.23 \pm 4.57$ & $47.75 \pm 8.56^{\mathrm{b}}$ \\
\hline \multicolumn{3}{|c|}{ Echocardiographic Parameters } \\
\hline IVST, mm & $9.38 \pm 0.92$ & $9.94 \pm 1.05$ \\
\hline LVPWT, mm & $9.42 \pm 0.85$ & $9.83 \pm 1.01$ \\
\hline LVEDD, mm & $43.21 \pm 5.44$ & $45.36 \pm 6.62$ \\
\hline LVEF,\% & $65.78 \pm 5.76$ & $63.47 \pm 6.02$ \\
\hline Dcs, cm & $5.75 \pm 1.14$ & $4.43 \pm 1.26^{\mathrm{b}}$ \\
\hline VTI, cm & $15.48 \pm 4.37$ & $13.48 \pm 2.19^{b}$ \\
\hline Flow, $\mathrm{mL} /$ minute & $306.78 \pm 120.81$ & $181.38 \pm 108.72^{b}$ \\
\hline LVM, M-mode g & $132.43 \pm 22.61$ & $163.67 \pm 37.79^{b}$ \\
\hline Flow/LVM, mL/minuteg & $2.51 \pm 1.43$ & $1.97 \pm 1.14^{\mathrm{b}}$ \\
\hline LVd Mass A-L, g & $128.93 \pm 31.78$ & $124.52 \pm 34.89$ \\
\hline LVs Mass A-L, g & $89.87 \pm 28.71$ & $101.97 \pm 30.34^{\mathrm{b}}$ \\
\hline LV(d-s) Mass A-L, g & $39.95 \pm 18.47$ & $22.91 \pm 17.14^{\mathrm{C}}$ \\
\hline
\end{tabular}

a Abbreviations; CAD, coronary artery disease; HR, heart rate; SBP, systolic blood pressure; DBP, diastolic blood pressure; PP, pulse pressure; IVST, diastolic interventricular septal thickness; LVPWT, diastolic posterior wall thickness; LVEDD, left ventricular end-diastolic diameter; LVEF, left ventricular ejection fraction; Dcs, the diameter of coronary sinus; VTI, velocity time integral; LVM(M- mode), left ventricular mass using M mode formulas calculation methods; LVd Mass A-L, left ventricular mass at end diastole using area-length calculation methods; LVs Mass A-L, left ventricular mass at peak systole using area-length calculation methods; $\mathrm{LV}(\mathrm{d}-\mathrm{s})$ Mass A-L, the difference of left ventricular mass at end diastole and peak systole using area-length calculation methods

$\mathrm{b}_{\mathrm{P}<0.05}$., unpaired t test, compared to the values of normal subjects

${ }^{\mathrm{C}} \mathrm{P}<0.01$, unpaired $\mathrm{t}$ test, compared to the values of normal subjects 
Table 2. Comparison of Clinical and Echocardiographic Parameters Between Patients with Single-Vessel, Double-Vessel and TripleVessel Coronary Artery Disease ${ }^{\mathrm{a}}$

\begin{tabular}{|c|c|c|c|}
\hline Parameters & Single-Vessel Patients $(\mathrm{n}=12)$ & Double-Vessel Patients $(n=16)$ & Triple-Vessel Patients $(\mathrm{n}=12)$ \\
\hline \multicolumn{4}{|l|}{ Clinical Parameters } \\
\hline HR, beats/min & $73.51 \pm 10.76$ & $73.54 \pm 11.09$ & $74.4 \pm 11.05$ \\
\hline SBP, mmHg & $123 \pm 17.79$ & $124 \pm 25.79$ & $124.58 \pm 26.37$ \\
\hline DBP, mmHg & $71.21 \pm 6.85$ & $70.21 \pm 6.64$ & $72.75 \pm 9.23$ \\
\hline $\mathrm{PP}, \mathrm{mmHg}$ & $52.23 \pm 7.96$ & $54.23 \pm 4.85$ & $53.75 \pm 8.98$ \\
\hline \multicolumn{4}{|c|}{ Echocardiographic Parameters } \\
\hline IVST, mm & $9.63 \pm 0.84$ & $9.67 \pm 0.99$ & $9.74 \pm 0.95$ \\
\hline LVPWT, mm & $9.54 \pm 0.88$ & $9.62 \pm 0.95$ & $9.76 \pm 0.71$ \\
\hline LVEDD, mm & $48.25 \pm 5.49$ & $47.29 \pm 5.66$ & $49.39 \pm 5.67$ \\
\hline LVEF, \% & $66.79 \pm 6.78$ & $65.78 \pm 5.76$ & $64.47 \pm 5.92$ \\
\hline Dcs, cm & $4.79 \pm 1.84$ & $4.66 \pm 1.54$ & $3.43 \pm 0.86^{\mathrm{b}}$ \\
\hline VTI, cm & $11.46 \pm 2.63$ & $12.13 \pm 2.65$ & $10.25 \pm 3.17^{\mathrm{b}}$ \\
\hline Flow, mL/minute & $256.68 \pm 112.87$ & $249.78 \pm 109.65$ & $193.59 \pm 76.74^{\mathrm{b}}$ \\
\hline LVM, M- Mode g & $135.45 \pm 20.59$ & $136.43 \pm 28.64$ & $137.97 \pm 29.75$ \\
\hline Flow/LVM, mL/minute g & $1.98 \pm 1.56$ & $1.97 \pm 1.53$ & $1.85 \pm 0.94^{b}$ \\
\hline LVd Mass A-L, g & $132.96 \pm 30.76$ & $129.93 \pm 32.77$ & $131.51 \pm 36.94$ \\
\hline LVs Mass A-L, g & $109.67 \pm 26.73$ & $107.87 \pm 28.71$ & $109.96 \pm 32.05$ \\
\hline LV(d-s) Mass A-L, g & $23.95 \pm 16.85$ & $22.95 \pm 19.42$ & $22.91 \pm 18.14$ \\
\hline
\end{tabular}

a Abbreviations: HR, heart rate; SBP, systolic blood pressure; DBP, diastolic blood pressure; PP, pulse pressure; IVST, diastolic interventricular septal thickness; LVPWT, left ventricular diastolic posterior wall thickness; LVEDD, left ventricular end-diastolic diameter; LVEF, left ventricular ejection fraction; Dcs, the diameter of coronary sinus; VTI, velocity time integral; LVM (M- mode), left ventricular mass using M mode formulas calculation methods; LVd Mass A-L, left ventricular mass at end diastole using area-length calculation methods; LVs Mass A-L, left ventricular mass at peak systole using area-length calculation methods; LV (d-s) Mass A-L, the difference of left ventricular mass at end diastole and peak systole using area-length calculation methods

${ }^{\mathrm{b}} \mathrm{P}<0.05$, unpaired $\mathrm{t}$ test, compared to the values of single-vessel and double-vessel CAD patients.

Table 3. Coronary Angiographic Findings in Patients with Coronary Artery Disease a

\begin{tabular}{|c|c|}
\hline Portion & Number of Lesions \\
\hline \multicolumn{2}{|l|}{ LAD } \\
\hline Proximal & 13 \\
\hline Middle & 27 \\
\hline Distal & 15 \\
\hline \multicolumn{2}{|l|}{ LCX } \\
\hline Proximal & 23 \\
\hline Middle & 11 \\
\hline Distal & 9 \\
\hline \multicolumn{2}{|l|}{ RCA } \\
\hline Proximal & 4 \\
\hline Middle & 10 \\
\hline Distal & 12 \\
\hline FDB & 11 \\
\hline
\end{tabular}

\footnotetext{
a Abbreviations: LAD, left anterior descending coronary artery; LCX, left circumflex artery; RCA, right coronary artery; FDB, the first diagonal branch
}

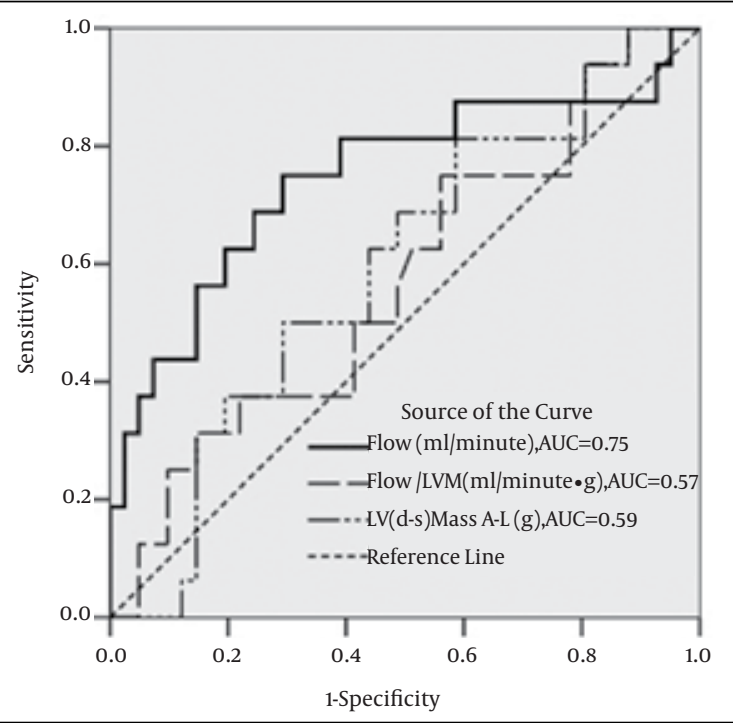

Figure 3. Receiver operating characteristic curve showing the performance of blood flow in the coronary sinus [flow $(\mathrm{mL} /$ minute)], the global left ventricular perfusion that was measured by dividing coronary sinus flow by the left ventricular mass [flow/LVM (mL/minuteg)] and the difference of left ventricular mass at end diastole and peak systole using arealength calculation methods [LV (d-s) mass A-L (g)] in discrimination between patients with or without CAD 
4.3. The Pearson Correlation Coefficient of Echocardiographic Parameters with a Percentage of Stenosis in Patients with Coronary Artery Disease

Flow (mL/minute), flow/LVM (mL/minuteg) and LV (d-s) mass A-L all correlated significantly with the percentage of stenosis $(\mathrm{r}=-0.64, \mathrm{P}<0.001 ; \mathrm{r}=-0.47, \mathrm{P}<0.001 ; \mathrm{r}=-0.56$, $\mathrm{P}<0.001$, respectively).

\subsection{ROC Analysis}

Using percentage of stenosis $>50 \%$ as the criteria to distinguish patients with or without $\mathrm{CAD}$, the areas under the ROC (AUC) for flow (mL/minute) were 0.75 , while they were 0.57 for flow/LVM (mL/minuteg) and 0.59 for $\mathrm{LV}(\mathrm{d}-\mathrm{s})$ mass A-L (g). The AUC for flow (mL/minute) was maximal, which was far higher than that of flow/LVM (mL/minuteg) and LV (d-s) mass A-L (g) $(\mathrm{P}<0.05)$ (Figure 3).

\subsection{Discriminant Analysis}

Based on the data depicted above, discriminant analysis of flow ( $\mathrm{mL} /$ minute) for predicting a significant coronary artery stenosis $(>70 \%)$ in patients with CAD was conducted. The best cutoff value was flow ( $\mathrm{mL} / \mathrm{minute})$ of less than $198 \mathrm{~mL} /$ minute) (sensitivity, 81.35\%; specificity, 70.37\%; positive predictive value, $63.63 \%$; negative predictive value, 86.36\%; and accuracy, $75 \% ; \mathrm{P}<0.001$ ).

\subsection{Reproducibility}

Intraobserver and interobserver variability for flow per minute were $7.3 \% \pm 2.6 \%$ and $7.9 \% \pm 2.4 \%$, respectively. Intraobserver and interobserver variability for LVM (Mmode) (g) were $2.5 \% \pm 0.9 \%, 2.9 \% \pm 1.2 \%$, respectively. Intraobserver and interobserver variability for LV(d-s) mass A-L (g) were $7.2 \% \pm 3.1 \%$ and $7.8 \% \pm 2.7 \%$, respectively.

\section{Discussion}

The results presented here indicate that the coronary sinus flow per minute is the most accurate parameter among the three echocardiographic parameters: the coronary sinus flow, the global left ventricular perfusion and the difference of left ventricular mass in the cardiac cycle, for assessment of significant stenosis of the coronary artery. A number of myocardial Doppler derived velocity, strain myocardial imaging parameters and speckle tracking imaging and wall motion score index have been proposed for the quantification of myocardial ischemia during stress echocardiography (12-15). But in the routine echocardiographic examination, they all have difficulties in the early detection of the presence of myocardial ischemia to some extent, especially when angina or myocardial infarction has not yet occurred. Although myocardial contrast echocardiography is an accurate imaging tool for the assessment of the myocardial microcirculation (16), it is time-consuming, expensive, and it is not always readily available. Alternative methods for the de- tection of myocardial ischemia are needed. The coronary sinus flow, the global left ventricular perfusion and the difference of left ventricular mass measured by ordinary two-dimensional and Doppler echocardiography, being rapid, convenient, inexpensive, reliable, and noninvasive in the assessment of significant stenosis of the coronary artery territory, has potential for clinical implications.

In our study, the average CS flow was determined to be $306.78 \pm 120.81 \mathrm{~mL} / \mathrm{min}$ in normal subjects and 181.38 \pm 108.72 in patients with CAD. This is in range with what has been found in previous echocardiographic studies, where values from $250 \mathrm{~mL} / \mathrm{min}$ (17) to $450 \mathrm{~mL} / \mathrm{min}$ (5) in normal subjects and from $150 \mathrm{~mL} / \mathrm{min}$ (18) to $250 \mathrm{~mL} / \mathrm{min}$ (17) in patients with CAD have been reported. The average value of $\mathrm{LV}$ perfusion, $2.51 \pm 1.43 \mathrm{~mL} /$ minuteg in normal subjects and $1.97 \pm 1.14 \mathrm{~mL} /$ minuteg in patients with CAD are significantly greater than other published values determined by magnetic resonance imaging, which lie between $0.60 \pm 0.22 \mathrm{~mL} / \mathrm{ming}$ and $0.53 \pm 0.14 \mathrm{~mL} / \mathrm{ming}$ ( 6 , 19-21). Maybe, the different imaging methods are accountable for these differences. The average value of the difference of left ventricular mass in the cardiac cycle, $39.95 \pm$ $18.47 \mathrm{~g}$ in normal subjects and $22.91 \pm 17.14 \mathrm{~g}$ in patients with $\mathrm{CAD}$, is also in accordance with published values, which is $40.12 \pm 14.37 \mathrm{~g}$ in normal subjects and $21.36 \pm 17.55$ $\mathrm{g}$ in patients with CAD (7). In our study group, flow (mL/ minute), flow/LVM (mL/minuteg) and LV (d-s) mass A-L all correlated significantly with the percentage of stenosis of the left coronary artery territory. Using a percentage of stenosis $>50 \%$ as the criteria to distinguish patients with or without $\mathrm{CAD}$, the areas under the ROC curve for flow (mL/minute) were 0.75, while they were 0.57 for flow/LVM (mL/minuteg) and 0.59 for LV (d-s) mass A-L. The AUC for flow ( $\mathrm{mL} /$ minute) was maximal, which indicated that flow ( $\mathrm{mL} /$ minute) has the greatest ability to distinguish patients with or without CAD. The following factors can account for this result. First, LVM as measured by M-mode and two-dimensional echocardiography has inherent defects, which assume left ventricular as a prolate ellipsoid shape (22). Formulas were developed for the calculation of LVM based on the regression equations of the calculated mass to autopsy findings. Some limits, such as non-standard left ventricular long or short axis view, unclear epicardial or endocardial boundaries, and the poor acoustic windows, all affect the accuracy of the measurements. However, coronary sinus can be adequately visualized from the parasternal right ventricular inflow tract view through TTE approach in almost all subjects, and the coronary sinus flow in this view can be monitored by TTE within a distance of 1 to $1.5 \mathrm{~cm}$ from the ostium with $\mathrm{a}<30^{\circ}$ Doppler angle between the ultrasound beam and the vessel. Although sometimes some limitations such as the interference of the heart movements and the poor acoustic windows also exist, the coronary sinus flow can more directly reflect the homodynamic changes of blood flow in the left coronary artery territory than the global left ventricular perfusion and the difference of left ven- 
tricular mass in the cardiac cycle. In this study, we ranked the echocardiographic parameters: the coronary sinus flow, the global left ventricular perfusion and the difference of left ventricular mass in cardiac cycle vs. coronary angiography as gold standard for the assessment of significant stenosis of the left coronary artery territory. We demonstrate that the coronary sinus flow per minute is the most accurate one among these parameters. Although some limitations have been mentioned above, this method still holds considerable clinical promise for the diagnosis of CAD.

\section{Acknowledgements}

The authors gratefully acknowledge the technical assistance and helpful discussion of Li J, Huang XQ, Fan GX at the department of ultrasound, The First People's Hospital of Yancheng, Jiangsu Province, P.R. China.

\section{Authors' Contributions}

Bin Yang and Xiao-ZhiZheng developed the original idea and the protocol, abstracted and analyzed the data, wrote the manuscript, and is guarantor. Jing Wu contributed to the development of the protocol, abstracted data, and prepared the manuscript.

\section{References}

1. Vrublevsky AV, Boshchenko AA, Karpov RS. Reduced coronary flow reserve in the coronary sinus is a predictor of hemodynamically significant stenoses of the left coronary artery territory. Eur JEchocardiogr. 2004;5(4):294-303.

2. Vrublevsky AV, Boshchenko AA, Karpov RS. Simultaneous transesophageal Doppler assessment of coronary flow reserve in the left anterior descending artery and coronary sinus allows differentiation between proximal and non-proximal left anterior descending artery stenoses. Eur JEchocardiogr. 2004;5(1):25-33.

3. Ramos Filho J, Ramires JA, Turina M, Medeiros CJ, Lachat M, Tsutsui J. Study of coronary sinus flow reserve through transesophageal Doppler echocardiography in normal subjects. Arq Bras Cardiol. 2002;79(2):97-106.

4. Hood WB, Jr. Regional venous drainage of the human heart. $\mathrm{Br}$ Heart J.1968;30(1):105-9.

5. Zheng X, Ji P, Mao H, Hu J. Reduced antegrade flow in the coronary sinus is a predictor of coronary artery stenosis in hypertensive patients. JUltrasound Med. 2012;31(1):7-14.

6. Bloch KM, Carlsson M, Arheden H, Stahlberg F. Quantifying coronary sinus flow and global LV perfusion at 3T. BMC Med Imaging. 2009;9:9.

7. Zheng XZ, Ji P, Mao HW. Reduced difference in left ventricular mass at end diastole and peak systole is a predictor of major stenosis of the left coronary artery territory. J Ultrasound Med. 2012;31(9):1437-42.

8. Devereux RB, Alonso DR, Lutas EM, Gottlieb GJ, Campo E, Sachs I, et al. Echocardiographic assessment of left ventricular hypertrophy: comparison to necropsy findings. Am J Cardiol. 1986;57(6):450-8.

9. Judkins MP. Selective coronary arteriography. I. A percutaneous transfemoral technic. Radiology. 1967;89(5):815-24.

10. Akalin EN, Yaylali O, Kirac FS, Yuksel D, Kilic M. The Role of Myocardial Perfusion Gated SPECT Study in Women with Coronary Artery Disease: A Correlative Study. Mol Imaging Radionucl Ther. 2012;21(2):69-74.

11. Petcherski O, Gaspar T, Halon DA, Peled N, Jaffe R, Molnar R, et al. Diagnostic accuracy of 256-row computed tomographic angiography for detection of obstructive coronary artery disease using invasive quantitative coronary angiography as reference standard. Am J Cardiol. 2013;111(4):510-5.

12. Celutkiene I, Zakarkaite D, Skorniakov V, Zvironaite V, Grabauskiene V, Burca J, et al. Quantitative approach using multiple single parameters versus visual assessment in dobutamine stress echocardiography. Cardiovasc Ultrasound. 2012;10:31.

13. Moonen M, Lancellotti P, Zacharakis D, Pierard L. The value of 2D strain imaging during stress testing. Echocardiography. 2009;26(3):307-14.

14. Thorstensen A, Amundsen BH, Dalen H, Hala P, Kiss G, Aase SA, et al. Strain rate imaging combined with wall motion analysis gives incremental value in direct quantification of myocardial infarct size. Eur Heart J Cardiovasc Imaging. 2012;13(11):914-21.

15. Valocik G, Valocikova I, Mitro P, Fojtikova L, Druzbacka L, Kristofova B, et al. Diagnostic accuracy of global myocardial deformation indexes in coronary artery disease: a velocity vector imaging study. Int J Cardiovasc Imaging. 2012;28(8):1931-42.

16. Lepper W, Belcik T, Wei K, Lindner JR, Sklenar J, Kaul S. Myocardial contrast echocardiography. Circulation. 2004;109(25):3132-5.

17. Liu ZB, Tan YP, Fang P, Song W, Wu SB, Cong Y. Detection and reproducibility of coronary sinus with transthoracic Doppler echocardiography. Chin J Med Imaging Technol. 2005;12(21):1888-90.

18. Fang P, Tan YP, Liu ZB, Wu SB, Cong Y. Observation of blood flow changes in coronary sinus by transthoracic echocardiography before and after coronary artery interventional therapy. Acad J Sec Mil Med Univ. 2007;28(3):335-6.

19. Schwitter J, DeMarco T, Kneifel S, von Schulthess GK, Jorg MC, Arheden $\mathrm{H}$, et al. Magnetic resonance-based assessment of global coronary flow and flow reserve and its relation to left ventricular functional parameters: a comparison with positron emission tomography. Circulation. 2000;101(23):2696-702.

20. Koskenvuo JW, Hartiala JJ, Knuuti J, Sakuma H, Toikka JO, Komu $\mathrm{M}$, et al. Assessing coronary sinus blood flow in patients with coronary artery disease: a comparison of phase-contrast MR imaging with positron emission tomography. AJR Am J Roentgenol. 2001;177(5):1161-6.

21. Koskenvuo JW, Sakuma H, Niemi P, Toikka JO, Knuuti J, Laine H, et al. Global myocardial blood flow and global flow reserve measurements by MRI and PET are comparable. JMagn Reson Imaging. 2001;13(3):361-6.

22. Myerson SG, Montgomery HE, World MJ, Pennell DJ. Left ventricular mass: reliability of M-mode and 2-dimensional echocardiographic formulas. Hypertension. 2002;40(5):673-8. 